\title{
Target Detection and Tracking Modeling of Fire-control Radar
}

\author{
Hao Xu, Wenhao Lian, Qiang Xiao, Qian Zheng, Zhenqin Fan
}

No. 63981 Unit of PLA, Wuhan 430311, China

\author{
Keywords: Fire-control Radar, Detection Tracking, Signal Leveling, Modeling.
}

\begin{abstract}
Target detection and tracking of fire-control radar is the primary task of air defend combat and a key of fire-control system. First, wording process of fire-control radar system is analyzed. Then signal simulation modeling and mathematical modules are proposed. Finally, signal simulation of dynamic target detection and tracking is verified by simulation. The results show that not only the efficiency of modeling method and modules, but also is of great reference value for simulation research of performance evaluation, data processing.
\end{abstract}

\section{Introduction}

As the key detection senser to determine the outcome of the ground air defence combat system, to detect target in a High-precision, tract continuous target and real-time automatic measurement of the target position is the most prominent feature and the most basic mission of the fire-control radar. Therefore, the target detection and tracking analysis of the fire-control radar has always been highly concerned at home and abroad.

With the rapid development of simulation technology, simulation technology has taken the place of traditional real-time testing as an effective analytical method and has been widely used in radar field. From the current research situation in our country, there are many literatures about fire control radar system modeling and simulation. But there are two main problems: First, at the signal level, only for the fire control radar subsystem [2-3], respectively, the signal processing system, angle tracking system modeling and simulation study and analysis, not involved in signal processing and tracking control system integration research; At the functional level, the literature [4] has completed the function simulation of the fire control radar system, which can realize the continuous target detection and tracking simulation. However, due to the large granularity of the model,

Its vividness and credibility are urgent problems to be solved. In view of the above problems, based on the analysis of the target detection and tracking process of the fire-control radar system, the integrated modeling of radar target signal generation, signal processing and tracking control is completed based on the signal flow and the model of each module is given. Finally, The combination of each module of the fire control radar closed-loop dynamics to complete the distance, azimuth, high and low angle three-dimensional probe tracking simulation, and the simulation results were analyzed.

\section{Fire-Control Radar Target Detection and Tracking Modeling and Analysis}

Usually fire control radar is used to lock a single target in the air for continuous precision measurement of the tracking radar, a typical fire control radar target detection and tracking workflow shown in Fig 1. It can be seen that the fire detection radar tracking process is a closed-loop tracking system involving receiver, signal processing and tracking control system. It includes three closedloop tracking loops: distance tracking, azimuth tracking and high and low angle tracking. Based on the signal flow, we must complete the signal processing and tracking control system integrated modeling in order to achieve target detection and tracking simulation, it can be abstractly extracted into three modules: echo signal generation module, receiving and signal processing module, tracking control system module. 


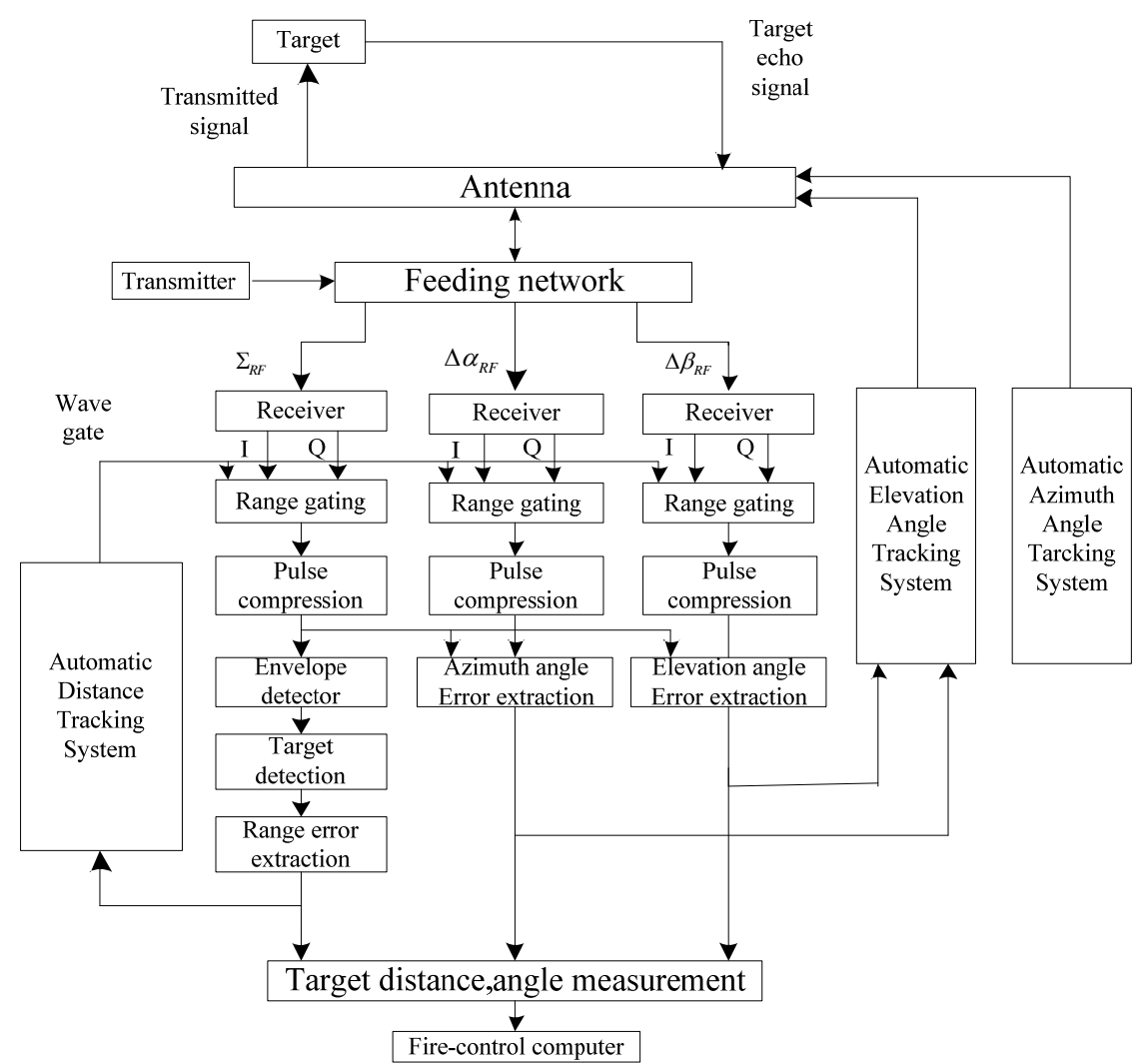

Fig. 1 A type of fire-control radar system target detection and tracking work flow chart

\section{Echo Signal Generation Module}

This paper focuses on the modeling of the target detection and tracking process. When the radar echo signal is simulated, the moving target echo signal and the thermal noise obeying Gaussian white noise distribution receiver are used to obtain the simulation.

\subsection{Antenna Pattern}

On the basis of the four feed beam patterns, the sum of the sum and difference beams of the radar through the antenna feed network can be described as follows: the sum of the signals, azimuths,

$$
\begin{aligned}
& F_{\Sigma}(\alpha, \beta)=F_{A}(\alpha, \beta)+F_{B}(\alpha, \beta)+F_{C}(\alpha, \beta)+F_{D}(\alpha, \beta) \\
& F_{\Delta \alpha}(\alpha, \beta)=F_{A}(\alpha, \beta)-F_{B}(\alpha, \beta)-F_{C}(\alpha, \beta)+F_{D}(\alpha, \beta) \\
& F_{\Delta \beta}(\alpha, \beta)=F_{A}(\alpha, \beta)+F_{B}(\alpha, \beta)-F_{C}(\alpha, \beta)-F_{D}(\alpha, \beta)
\end{aligned}
$$

\subsection{Modeling of Target Sum and Difference Echo Signal}

The essence of the radar signal is transmitted in the time domain, frequency domain, amplitude domain modulation, etc., on the basis of the sum and difference after the transformation will form a sum signal, azimuth difference signal, height difference signal, respectively, can be expressed as:

$$
\begin{aligned}
& \psi_{\Sigma}(t)=K G_{\Sigma} u\left(t-\tau_{0}\right) \exp \left[j 2 \pi\left(f_{0}+f_{d}\right)\left(t-\tau_{0}\right)+j k \pi\left(t-\tau_{0}\right)^{2}\right] \\
& \psi_{\alpha}(t)=K G_{\alpha} u\left(t-\tau_{0}\right) \exp \left[j 2 \pi\left(f_{0}+f_{d}\right)\left(t-\tau_{0}\right)+j k \pi\left(t-\tau_{0}\right)^{2}\right] \\
& \psi_{\beta}(t)=K G_{\beta} u\left(t-\tau_{0}\right) \exp \left[j 2 \pi\left(f_{0}+f_{d}\right)\left(t-\tau_{0}\right)+j k \pi\left(t-\tau_{0}\right)^{2}\right]
\end{aligned}
$$

Wherein, $u(t)$ is the complex modulation envelope, $f_{0}$ is the carrier center frequency, $T_{p}$ is the pulse width, $T_{r}$ is the pulse repetition period, B is the bandwidth of the chirp signal, $G$ is the gain of the antenna for the real-time antenna pattern pointing to, $k=B / T_{p}$ is the frequency modulation slope, $\mathrm{K}$ is the two-way attenuation factor of the signal after spatial propagation attenuation, $f_{d}$ is the Doppler shift of the relative motion between the target and the radar, $\tau_{0}$ is the time delay of the relative distance between the radar and the target. 


\section{Receive and Signal Processing Module}

\subsection{Receiver Model}

The receiver is three branch single pulse tracking receiver, after receiving three echo signals, to complete the process of down-conversion. Here, given the key aspects of coherent detection processing model, usually using orthogonal dual-channel detection method, the block diagram shown in Fig 2.

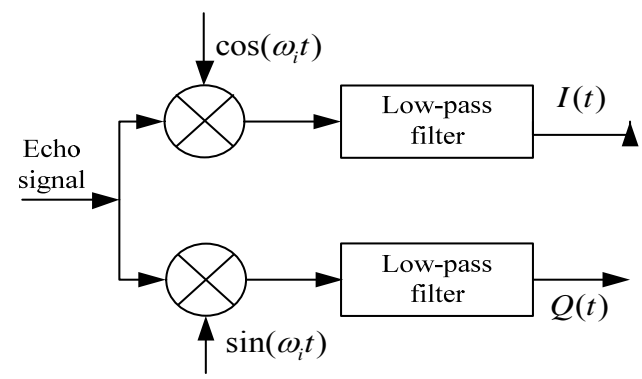

Fig 2 Simulation orthogonal two-channel detection block diagram

Since the principle of detection is the same, take the sum signal as an example, the in-phase branch $\Sigma_{I}$ and the orthogonal branch $\Sigma_{Q}$ is expressed as:

\subsection{Target Detection Model}

$$
\begin{aligned}
& \Sigma_{I}=K G_{\Sigma} u\left(t-\tau_{0}\right) \cos \left[k \pi\left(t-\tau_{0}\right)^{2}+2 \pi f_{d}\left(t-\tau_{0}\right)\right] \\
& \Sigma_{Q}=K G_{\Sigma} u\left(t-\tau_{0}\right) \sin \left[k \pi\left(t-\tau_{0}\right)^{2}+2 \pi f_{d}\left(t-\tau_{0}\right)\right]
\end{aligned}
$$

In this case, the probability of the receiver thermal noise envelope exceeding the detector threshold is the false alarm probability. In the case of ensuring the false alarm probability constant, then the detector threshold is:

$$
S=2 \sqrt{\frac{1}{\pi} \ln \left(1 / p_{f}\right)} E(v)
$$

Therein, $E(v)$ is the receiver thermal noise envelope detection amplitude average.

\subsection{Distance Error Extraction}

Usually in the distance tracking, the use of split-gate method, based on before and after the gate difference to complete the distance error extraction. In the simulation, the need to wait for the gating within the interval of the gate to carry out equal-interval sampling and related processing to distance measurement. Set the sampling numbers for the front and rear wave gates to be $\mathrm{N}$, the effective range of the wave gate unit expressed as, respectively, expressed as, at this time, the gate center and the target distance error can be based on the effective front and rear doors The difference between the distance of the unit and the sum of the range of the unit of the maximum effective distance within the range of the wave gate can be obtained by normalization. The distance error can be expressed as [7]:

$$
\Delta R=\frac{\sum_{i=1}^{N} H_{i}-\sum_{j=1}^{N} L_{j}}{2 K_{T} \max \left(H_{1}, H_{2} \ldots H_{N}, L_{1}, L_{2} \ldots L_{N}\right)}
$$

Wherein, for the time identification function slope

\subsection{Angle Error Extraction}

The target azimuth deviation angle and height deviation angle can be approximately estimated as:

$$
\begin{gathered}
\hat{\alpha}=\frac{1}{K_{\alpha}} \frac{\Sigma U_{\alpha}\left(\mathrm{N}_{\mathrm{i}}, \alpha\right)}{\Sigma U_{\Sigma}\left(\mathrm{N}_{\mathrm{i}}\right)} \\
\hat{\beta}=\frac{1}{\mathrm{~K}_{\beta}} \frac{\Sigma U_{\beta}\left(\mathrm{N}_{\mathrm{i}}, \beta\right)}{\Sigma U_{\Sigma}\left(\mathrm{N}_{\mathrm{i}}\right)}
\end{gathered}
$$

\section{Tracking Control System Module}

\subsection{Distance Tracking System Model}

In the simulation, the real-time target distance can be calculated by adding the distance unit of the gated FBG and the distance error, which can be expressed as: 


\subsection{Angle Tracking System Model}

$$
R=R_{\text {Gate center }}+\Delta R
$$

In practice, the azimuth and elevation measurements are output by an angle sensor that is directly mechanically coupled to the pedestal orientation and the high and low shaft systems.In the simulation, the azimuth and elevation measurements are calculated by superimposing the current pointing angle and the corresponding angular error of the radar antenna.

$$
\begin{aligned}
& \alpha=\alpha_{\text {Real-time direction }}+\hat{\alpha} \\
& \beta=\beta_{\text {Real-time direction }}+\hat{\beta}
\end{aligned}
$$

\section{Conclusion}

According to the typical single pulse radar fire control system, based on the analysis of target detection and tracking process, the fire control radar tracking process is divided into three simulation module, modeling of each module in the signal level, realize the fire control radar, the echo signal from the receiving, processing and automatic tracking system integration of dynamic closed-loop target the detection and tracking simulation modeling. This paper focuses on completing the whole process model and construction of the whole fire control radar's dynamic target detection and tracking. It will be simulated in future research, and the results will have some reference value for radar system design, test and evaluation.

\section{References}

[1]. Li, Jiang Liubing. Realization of ranging and velocity [J]. Modern radar software precision tracking radar, Vol.30 (2008), No.4, p. 56- 58.

[2]. David Adamy. A First Course of Electronic Warfare [M]. Beijing: Publish House of Electronic Industry, (2010).

[3]. Wang Xiang, Li Dun et al. Research on Modeling and Simulation of coherent video signal in phased array radar, [J]. System simulation, Vol.22 (2010), No.3, p. 741-747.

[4]. Mou Zelei. Simulation of a certain type of fire control radar signal processing [D]. Electronic Science and Technology University Master's degree thesis. (2011)

[5]. Yang Haiping. Design of the servo control system for fire control radar [D]. Nanjing University of Aeronautics \& Astronautics. (2010) 